COCHRANE CORNER

\footnotetext{
${ }^{\dagger}$ This review is an abridged version of a Cochrane review previously published in the Cochrane Database of Systematic Reviews, 2016, Apr 13, Issue 4: CD011004 (see www. cochranelibrary.com for information). Cochrane reviews are regularly updated as new evidence emerges and in response to feedback, and the Cochrane Database of Systematic Reviews should be consulted for the most recent version of the review.
}

We thank the Cochrane Review Group for their support in publishing these reviews.

See commentary on pp. $3-8$, this issue.

\title{
Psychological therapies for panic disorder with or without agoraphobia in adults: a network meta-analysis ${ }^{\dagger}$
}

\author{
Alessandro Pompoli, Toshi A Furukawa, Hissei Imai, Aran Tajika, Orestis Efthimiou \& Georgia Salanti
}

\section{Background}

Panic disorder is characterised by the presence of recurrent unexpected panic attacks, discrete periods of fear or anxiety that have a rapid onset and include symptoms such as racing heart, chest pain, sweating and shaking. Panic disorder is common in the general population, with a lifetime prevalence of $1-4 \%$. A previous Cochrane meta-analysis suggested that psychological therapy (either alone or combined with pharmacotherapy) can be chosen as a first-line treatment for panic disorder with or without agoraphobia. However, it is not yet clear whether certain psychological therapies can be considered superior to others. To answer this question, in this review we performed a network meta-analysis (NMA), in which we compared eight different forms of psychological therapy and three forms of a control condition.

\section{Objectives}

To assess the comparative efficacy and acceptability of different psychological therapies and control conditions for panic disorder, with or without agoraphobia, in adults.

\section{Search methods}

We conducted the main searches in the CCDANCTR electronic databases (studies and references registers), all years to 16 March 2015, and complementary searches in PubMed and trials registries. Supplementary searches included reference lists of included studies, citation indexes, personal communication to the authors of all included studies and grey literature searches in OpenSIGLE. We applied no restrictions on date, language or publication status.

\section{Selection criteria}

We included all relevant randomised controlled trials (RCTs) focusing on adults with a formal diagnosis of panic disorder with or without agoraphobia. We considered the following psychological therapies: psychoeducation (PE), supportive psychotherapy (SP), physiological therapies (PT), behavioural therapy (BT), cognitive therapy (CT), cognitive-behavioural therapy (CBT), third-wave CBT (3W) and psychodynamic therapies (PD). We included both individual and group formats. Therapies had to be administered faceto-face. The comparator interventions considered were: no treatment (NT), waiting list (WL) and attention/psychological placebo (APP). We considered four short-term (ST) outcomes (ST-remission, ST-response, ST-drop-out, ST-improvement on a continuous scale) and one long-term (LT) outcome (LT-remission/response).

Data collection and analysis

We conducted a systematic search of all relevant papers according to the inclusion criteria. For each outcome, we then constructed a treatment network to clarify the extent to which each type of therapy and each comparison had been investigated in the available literature. Then, for each available comparison, we conducted a random-effects meta-analysis. Subsequently, we performed a network meta-analysis to synthesise the available direct evidence with indirect evidence, and to obtain an overall effect size estimate for each possible pair of therapies in the network. Finally, we calculated a probabilistic ranking of the different psychological therapies and control conditions for each outcome.

\section{Main results}

We identified 1432 references; after screening, we included 60 studies in the final qualitative analyses. Among these, $54(N=$ 3021 patients) were also included in the quantitative analyses. In the analyses for the first of our primary outcomes (shortterm remission) the most studied of the included psychological therapies was CBT (32 studies), followed by BT (12 studies), PT (10 studies), CT (three studies), SP (three studies) and PD (two studies). The quality of the evidence for the entire network was found to be low for all outcomes. The quality of the evidence for CBT v. NT, CBT v. SP and CBT v. PD was low to very low, depending on the outcome. The majority of the included studies were at unclear risk of bias with regard to the randomisation process. We found almost half of the included studies to be at high risk of attrition bias and detection bias. We also found selective outcome reporting bias to be present and we strongly suspected publication bias. Finally, we found almost half of the included studies to be at high risk of researcher allegiance bias. Overall, the networks appeared to be well connected, but were generally underpowered to detect any important disagreement between direct and indirect evidence. The results showed the superiority of psychological therapies over the WL condition, although this finding was amplified by evident small study effects (SSE). The NMAs for ST-remission, ST-response and ST-improvement on a continuous scale showed well-replicated evidence in favour of $\mathrm{CBT}$, as well as some sparse but relevant evidence in favour of PD and SP, over other therapies. In terms of ST-drop-out, PD and $3 \mathrm{~W}$ showed better tolerability over other psychological therapies in the short term. In the long term, CBT and PD showed the highest level of remission/response, suggesting that the effects of these two treatments may be more stable than other psychological therapies. However, all the mentioned differences among active treatments must be interpreted while taking into account that in most cases the effect sizes were small and/or results were imprecise.

\section{Authors' conclusions}

There is no high-quality, unequivocal evidence to support one psychological therapy over the others for the treatment of panic disorder with or without agoraphobia in adults. However, the results show that CBT (the most extensively studied among the included psychological therapies) was often superior to other therapies, although the effect size was small and the level of precision was often insufficient or clinically irrelevant. In the only two studies that explored PD, this treatment showed promising results, although further research is needed to better explore its relative efficacy with respect to CBT. Furthermore, PD appeared to be the best tolerated (in terms of ST-drop-out) psychological treatment. Unexpectedly, we found some evidence in support of the possible viability of non-specific supportive psychotherapy for the treatment of panic disorder; however, the results concerning SP should be interpreted cautiously because of the sparsity of evidence regarding this treatment and, as in the case of PD, further research is needed to explore this issue. Behavioural therapy did not appear to be a valid alternative to CBT as a first-line treatment for panic disorder with or without agoraphobia. 